\title{
Overexpression of the Aryl Hydrocarbon Receptor (Ahr) Mediates an Oxidative Stress Response following Injection of Fine Particulate Matter in the Temporal Cortex
}

\author{
So Young Kim $\left(\mathbb{D},{ }^{1}\right.$ Kyung Woon Kim $\mathbb{D D}^{1},{ }^{1}$ So Min Lee $\left(\mathbb{D},{ }^{1}\right.$ Da-hye Lee $(\mathbb{D}),{ }^{1}$ Sohyeon Park, ${ }^{2}$ \\ Bu Soon Son, ${ }^{2}$ and Moo Kyun Park $\mathbb{1}^{3,4}$ \\ ${ }^{1}$ Department of Otorhinolaryngology, CHA University College of Medicine, Seongnam, Republic of Korea \\ ${ }^{2}$ Department of Medical Biotechnology, SoonChunHyang University, Asan, Chungnam, Republic of Korea \\ ${ }^{3}$ Department of Otorhinolaryngology, Seoul National University College of Medicine, Seoul, Republic of Korea \\ ${ }^{4}$ Sensory Organ Research Institute, Seoul National University Medical Research Center, Seoul, Republic of Korea
}

Correspondence should be addressed to Moo Kyun Park; entpmk@gmail.com

Received 15 July 2020; Revised 10 November 2020; Accepted 16 December 2020; Published 28 December 2020

Academic Editor: Gabriele Saretzki

Copyright (C) 2020 So Young Kim et al. This is an open access article distributed under the Creative Commons Attribution License, which permits unrestricted use, distribution, and reproduction in any medium, provided the original work is properly cited.

\begin{abstract}
Studies have shown that particulate matter (PM) induces the expression of the aryl hydrocarbon receptor (Ahr) leading to the activation of the oxidative stress response. This study is aimed at characterizing the specific impact of fine PM on the expression profile of the Ahr and oxidative stress response in the primary auditory cortex. $\mathrm{PM}_{2.5}(<1.8 \mu \mathrm{m})$-loaded filters were suspended in sterile saline to $102.6-111.82 \mu \mathrm{g} / \mathrm{ml}$. Next, $10 \mu \mathrm{l}$ of $\mathrm{PM}_{2.5}$ or an equal volume of saline was administered intracranially into the temporal cortex of two groups of rats $\left(\mathrm{PM}_{2.5}\right.$ and control; $n=14$ per group), respectively. One week after intracranial injection, the temporal cortex was harvested. Transmission electron microscopy was performed to evaluate the distribution of $\mathrm{PM}_{2.5}$ within the temporal cortex. Additionally, the mRNA and protein expression levels of cytochrome P450 1A1 (CYP1A1), CYP1B1, inducible nitric oxide synthase (iNOS), Ahr, and brevican mRNA and protein were measured using quantitative reverse transcription-polymerase chain reaction (qRT-PCR) or western blotting, respectively. Finally, the protein expression levels of the receptor for advanced glycation end products (RAGE) were estimated using enzyme-linked immunosorbent assay (ELISA). $\mathrm{PM}_{2.5}$ was observed in intracellular vesicles within the temporal cortex following intracranial injection. Levels of oxidative stress molecules (i.e., CYP1A1, CYP1B1, and iNOS), Ahr, Brevican, and RAGE were higher in the $\mathrm{PM}_{2.5}$ group compared with the control group. Intracranial administration of $\mathrm{PM}_{2.5}$ led to increased levels of Ahr and markers of an oxidative stress response in the temporal cortex. The oxidative stress response-mediated increases in the levels of brevican and RAGE.
\end{abstract}

\section{Introduction}

Particulate matter (PM) has been suggested to have adverse effects on the central nervous, respiratory, and cardiovascular systems. In a clinical study involving subjects from the United States, long-term exposure to $\mathrm{PM}_{2.5}$ (fine particles $<2.5 \mu \mathrm{m}$ ) and $\mathrm{PM}_{10}$ (inhalable particles $<10 \mu \mathrm{m}$ ) was associated with reduced brain volume [1]. A number of other studies have described CNS injuries after inhalation of PMs [2-5]. In a rat study, long-term (i.e., 3 and 6 months) exposure to $\mathrm{PM}_{1}$ induced a short-term memory deficit, spongiosis, and neuronal shrinkage and led to increases in proinflammatory molecules including interleukin (IL) 6 in the hippocampus [2]. In a mouse inhalation study, inhalation of ambient PM for 4 weeks led to (i) an upregulation of proinflammatory molecules, (ii) downregulation of molecules of the brain-derived neurotrophic factor (BDNF) signaling pathway (e.g., BDNF, high-affinity receptor tropomyosin-related kinase B (TrkB), cyclic adenosine monophosphate-response element-binding protein), and (iii) depressive-like behavioural changes [3]. Previous studies by this group have also demonstrated proinflammatory and oxidative stress responses, alterations in the expression of vesicular synaptic transporters, and perineuronal changes following 4-week and 8-week exposures to $\mathrm{PM}_{10}$ in cerebral cortical areas $[4,5]$. 
Systemic cardiovascular circulation and inhalation through the olfactory bulb cleft might be a primary route for PMs to reach the CNS in inhalation models [6]. Cerebral blood circulation might preferentially lead to an effect on brain regions with high metabolic rates; therefore, areas like the cortex might be more susceptible to chronic DEP inhalation-induced oxidative stress and inflammation. In line with this, previous animal inhalation studies reported more cerebral cortical inflammation and oxidative stress injuries compared with olfactory bulb injuries [3-5]. Relating to the diffusion of inhaled PMs via the nasal olfactory system, a number of previous studies reported olfactory deposition and translocation to the olfactory bulb [7-10]. Direct PM exposures such as those described above could lead to penetration and destruction of cellular integrity [11]. An in vitro study described the reduction of cellular integrity by transepithelial electric resistance following 24-hour exposure to commercial cigarette smoke condensates in brain tissue and astrocytes in the presence of a blood-brain barrier [11]. However, the direct penetrating effect of PM on the CNS has not been explored in vivo.

The aryl hydrocarbon receptor (AhR) has been proposed as a signal transducer of $\mathrm{PM}$-induced oxidative stress and inflammation [12, 13]. AhR is a basic helixloop-helix PER/ARNT/SIM family of transcription factors, which is activated by several exogenous ligands (e.g., polycyclic aromatic hydrocarbons and halogenated aromatic hydrocarbons) [14]. When $A h R$ ligands activate the $A h R$ and Aryl hydrocarbon receptor nuclear translocator, they bind to consensus xenobiotic responsive elements (XRE) located upstream of target genes (e.g., cytochromes P450 such as CYP1A1) [15]. Several published studies have reported $A h R$ activation in PM-induced respiratory or cardiovascular diseases [16, 17]. However, the molecular mechanisms of PM-mediated AhR activation in the CNS have not been well characterized.

This study hypothesized that the direct effects of $\mathrm{PM}_{2.5}$ on the cerebral cortex might be accompanied by the intracellular translocation of $\mathrm{PM}_{2.5}$ and that the proinflammatory and oxidative stress responses could be mediated by increases in $A h R$. To investigate the direct impact of $\mathrm{PM}_{2.5}$ on the temporal cortex in vivo, $\mathrm{PM}_{2.5}$ was injected intracranially in adult rats. The presence of $\mathrm{PM}_{2.5}$ in the temporal cortex was confirmed, and downstream response genes of CYP1A1 and CYP1B1 and inflammatory molecules were evaluated.

\section{Methods}

2.1. Animal Experiments. The study was approved by the Institutional Animal Care and Use Committee of CHA University Medical School (IACUC170162). Postnatal 8week-old 10 female and 4 male Sprague-Dawley rats were divided into $\mathrm{PM}_{2.5}$ and saline groups ( $n=14$ per group) (Figure 1). The $\mathrm{PM}_{2.5}$ rats received intracranial injections of $10 \mu \mathrm{l}$ of $102.6-111.82 \mu \mathrm{g} / \mathrm{ml} \mathrm{PM}_{2.5}$ (diameter $<1.8 \mu \mathrm{m}$ ) in the primary temporal cortex. Under anesthesia using a mixture of Zoletil $(40 \mathrm{mg} / \mathrm{kg})$ and xylazine $(10 \mathrm{mg} / \mathrm{kg})$, a $10 \mu \mathrm{l} 33$-gauge needle Hamilton syringe was used for the intracranial injections with a stereotactic apparatus as previously described [18]. The injection site was as follows (in $\mathrm{mm}$ from bregma and the skull): anterior posterior (AP): $-5 \mathrm{~mm}$, lateral (L): $7 \mathrm{~mm}$, ventral $(\mathrm{V}): 4.2 \mathrm{~mm}$ according to the atlas of Paxinos and Watson (2006). After the cortical bone was drilled, the meninges were punched with a sterile needle. The total injection volume of $10 \mu \mathrm{l}$ was infused with a speed of $0.2 \mu \mathrm{l} / \mathrm{min}$ using an automated microinjector (Harvard Apparatus, Saint-Laurent, QC, Canada), and the injected needle was maintained for 50 minutes [19-21]. In the saline group $(n=10), 10 \mu \mathrm{l}$ of saline was similarly injected into the primary temporal cortex. No rats died after intracranial injection, and no differences in weight and activities between $\mathrm{PM}_{2.5}$ and control groups were observed. All rats were sacrificed, and ipsilateral (intracranial injected site, right) and contralateral (left) temporal cortices were harvested as frozen tissue in 18 rats ( $n=9$ for each group). Whole brain tissue was harvested from the remaining 6 rats for histologic examination and immersion-fixed in $4 \%$ paraformaldehyde solution.

2.2. Fine Particulate Matters. Fine particulate matters were collected in an industrial region (Asan, Korea) five times between September 15, 2017, and November 21, 2017, using a previously described process [22]. The collected airborne fine particles were filtered on Teflon (PTFE) filters (Whatman, Maidstone, Kint, UK). After drying the filters in a desiccator for 48 hours, the weights of $\mathrm{PM}_{2.5}$ were measured using a microbalance (CP2P-F, Satorius, Coettingen, Germany). After baking for 2 hours, the filters were immersed in deionized water and sonicated for 1 hour. The compositions of heavy metals in $\mathrm{PM}_{2.5}$ were analyzed and are presented in Table 1.

2.3. Transmission Electron Microscopy. To characterize the localization of the injected $\mathrm{PM}_{2.5}$ in the temporal cortex, brain sections of $\mathrm{PM}_{2.5}$ rats were examined using transmission electron microscopy (TEM) [23]. The immersion-fixed brain samples were washed using deuterated $\mathrm{H}_{2} \mathrm{O}_{2}$ and dehydrated using ethanol solutions. The samples were infiltrated by propylene oxide and EPON epoxy resin mixed (Embed 812 , Nadic methyl anhydride, poly Bed 812 , dodecenylsuccinic anhydride, and dimethylaminomethyl phenol) (Electron Microscopy Polysciences, USA). The epoxy resin-embedded brains were polymerized at $38^{\circ} \mathrm{C}$ for 12 hours and $60^{\circ} \mathrm{C}$ for 48 hours. Using an ultramicrotome (RMC MT-XL), samples were thinly sectioned (roughly $65 \mathrm{~nm}$ ). Sections were then stained with saturated $4 \%$ uranyl acetate and $4 \%$ lead citrate. Finally, tissues were examined by TEM (JEM-1400, Japan) at $80 \mathrm{kV}$.

2.4. Expression Levels of Cytochrome P450 (CYP) Enzymes and Aryl Hydrocarbon Receptor (AhR). Real-time reverse transcription-polymerase chain reaction (RT-PCR) was performed using micropunched primary temporal cortex tissue as previously detailed [4]. Reverse transcription was performed using TOPreal ${ }^{\mathrm{TM}}$ qPCR 2X PreMIX' (Enzynomics, \#RT500M) and forward and reverse 


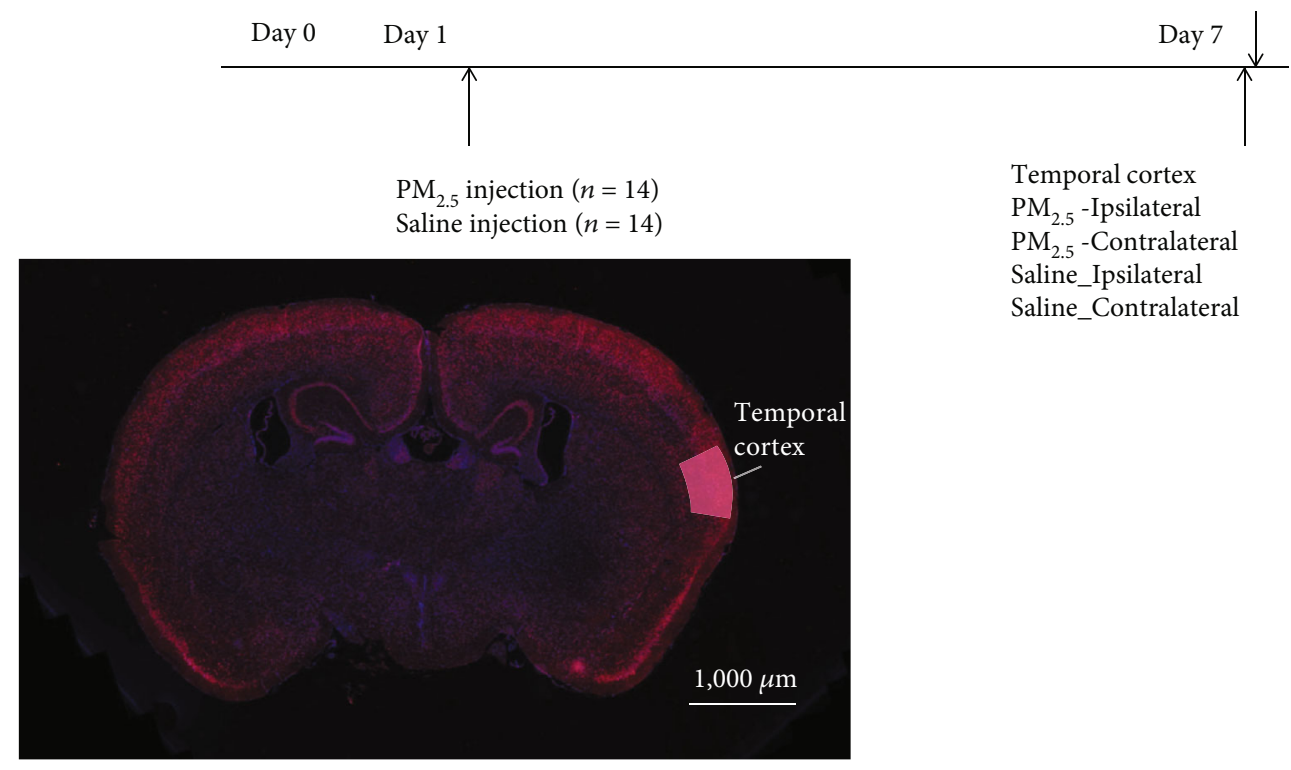

Figure 1: The experimental schedule of the present study. Postnatal 8-week-old female Sprague-Dawley rats received intracranial injections of $\mathrm{PM}_{2.5}$ (particulate matter diameter $<1.8 \mu \mathrm{m}$ ) or saline in the primary temporal cortex. The injection site of the temporal cortex was indicated with a red arrow.

TABLE 1: Compositions of heavy metals in fine particulate matters $\left(\mathrm{PM}_{2.5}\right)$.

\begin{tabular}{|c|c|c|c|c|c|c|c|c|}
\hline Heavy metals & $\mathrm{Cd}$ & As & $\mathrm{Pb}$ & $\mathrm{Cr}$ & $\mathrm{Cu}$ & $\mathrm{Mn}$ & $\mathrm{Ni}$ & $\mathrm{Be}$ \\
\hline Average concentrations (pg/2.72 $\mu \mathrm{g})$ & 3.17 & 21.42 & 46.73 & 21.49 & 104.53 & 108.06 & 7.29 & 0.00 \\
\hline Standard deviation & 2.11 & 13.48 & 30.15 & 22.67 & 34.55 & 33.94 & 3.83 & 0.00 \\
\hline
\end{tabular}

oligonucleotides for PCR amplification of cytochrome P450 (CYP)1A1, CYP1B1, inducible nitric oxide synthase (iNOS), and AhR (Table 2) using CFX connect $^{\mathrm{TM}}$ Realtime system (Bio-Rad, \#1855201). mRNA levels were normalized to glyceraldehyde 3-phosphate dehydrogenase.

2.5. Changes in the Expression Levels of AhR Protein in the Primary Temporal Cortex. Immunofluorescence staining was performed as previously described [4]. Specimens were dehydrated and embedded in paraffin with optimal cutting temperature solution. The $10 \mu \mathrm{m}$ sections of embedded tissue were cut on a rotary microtome and mounted on glass slides. Each slide was dipped in xylene for paraffin removal for $10 \mathrm{~min}$ and sequentially washed in $100 \%, 75 \%$, and $50 \%$ ethanol for $5 \mathrm{~min}$ each wash. The free-floating sections were then washed in PBS three times for $5 \mathrm{~min}$ per wash. After a set of three $5 \mathrm{~min}$ washes in PBS, sections were placed in $10 \%$ goat or donkey blocking serum (Vector Labs, Burlingame, CA, USA) for 1 hour at room temperature. Free-floating slices were then incubated overnight at $4^{\circ} \mathrm{C}$ on a shaking table with an antiAhr primary antibody (mouse monoclonal, Santa Cruz Biotechnology). The following day, slices were washed in PBS three times for $10 \mathrm{~min}$ per wash. Then, sections were incubated with secondary antibody (Alexa Fluor 594 donkey anti-mouse (Invitrogen, USA, \#A21203)) for 2 hours at room temperature. The primary auditory cortex (A1) areas from each hemisphere were analyzed: AP: $-5 \mathrm{~mm}$,
$\mathrm{L}: 7 \mathrm{~mm}$, and $\mathrm{V}: 3.1-4.9 \mathrm{~mm}$. Images were photographed using a fluorescence microscope (ECLIPSE Ni-U, Nikon Corporation, Japan).

2.6. Protein Expression Levels of Matrix Metalloproteinase (MMP) 9 and Brevican. Western blotting was performed as previously described [4]. Approximately $20 \mu \mathrm{g}$ of protein was separated by $12 \%$ sodium dodecyl sulphatepolyacrylamide gel electrophoresis (SDS-PAGE) and transferred to polyvinylidene difluoride (PVDF) membranes (Merck Millipore, Burlington, MA, USA). Membranes were soaked in blocking buffer ( $5 \%$ nonfat dry milk in Trisbuffered saline containing Tween-20 [TBS-T]) for 1 hour at room temperature followed by incubation with specific primary antibodies: anti-MMP9 (rabbit monoclonal, Abcam), antibrevican (rabbit polyclonal, Abcam), and $\beta$-actin (D6A8, rabbit mAb; Cell Signaling Technology). Immunoreactive proteins were detected with a horseradish peroxidase(HRP-) coupled secondary antibody (anti-rabbit IgG, HRPlinked antibody; Cell Signaling Technology) and visualised using an enhanced chemiluminescence (ECL) kit (Bio-Rad). Protein bands were quantitated by densitometry using ImageJ gel analysis software (National Institutes of Health, Bethesda, MD, USA). The protein expression levels were normalized to $\beta$-actin.

2.7. Protein Expression Level of RAGE. The levels of RAGE protein were determined by enzyme-linked immunosorbent 
TABLE 2: Oligonucleotide primer sequences for quantitative reverse transcriptase-polymerase chain reaction.

\begin{tabular}{|c|c|c|c|c|c|}
\hline Gene & Primer sequence (forward) & Primer sequence (reverse) & $\begin{array}{c}\text { Annealing } \\
\text { temperature }\left({ }^{\circ} \mathrm{C}\right)\end{array}$ & $\begin{array}{l}\text { Product size } \\
\text { (bp) }\end{array}$ & $\begin{array}{c}\text { RefSeq } \\
\text { number }\end{array}$ \\
\hline CYP1A1 & $\begin{array}{c}5^{\prime} \text {-CATCCCCCACAGCACC } \\
\text { ATAA- }{ }^{\prime}\end{array}$ & $\begin{array}{c}5^{\prime} \text {-TTCGCTTGCCCAAACCAAAG- } \\
3^{\prime}\end{array}$ & 60 & 212 & $\begin{array}{l}\mathrm{NM}_{-} \\
012540.2\end{array}$ \\
\hline CYP1B1 & $\begin{array}{c}5^{\prime} \text {-TGCTACTCGTTTCGGT } \\
\text { CCTG-3' }\end{array}$ & $\begin{array}{c}5^{\prime} \text {-CAAGGCGAGCGAAGTA } \\
\text { CAAG- } 3^{\prime}\end{array}$ & 60 & 162 & $\mathrm{NM}_{0}$ \\
\hline iNOS & $\begin{array}{c}5^{\prime} \text {-AGGCCACCTCGGATATCTC } \\
\text { T- } 3^{\prime}\end{array}$ & $\begin{array}{c}5^{\prime} \text {-TCTCTGGGTCCTCTGGTCAA- } \\
3^{\prime}\end{array}$ & 60 & 85 & $\underset{012611.3}{\mathrm{NM}_{-}}$ \\
\hline$A h R$ & $\begin{array}{c}5^{\prime} \text {-CTCCCTCCACAGTTGGCTT } \\
\text { TGTTTG-3' }\end{array}$ & $\begin{array}{c}5^{\prime} \text {-GATTCTGCGCAGTGAAGCA } \\
\text { TGTCAG-3' }\end{array}$ & 60 & 233 & $\begin{array}{c}\mathrm{NM}_{-} \\
013149.3\end{array}$ \\
\hline
\end{tabular}

assay (ELISA). We used the corresponding quantitative rat Immunoassay ELISA (ab202409, Abcam) according to the manufacturer's instructions. Briefly, absorbance was measured spectrophotometrically at $450 \mathrm{~nm}$ (SpectraMax M2, Molecular Devices, USA). The temporal cortex was homogenized with PBS ( $\mathrm{pH} 7.4$ ) solution containing 1\% Triton X100 and then centrifuged at $3000 \mathrm{rpm}$ for $15 \mathrm{~min}$. The supernatant was collected and immediately stored at $-80^{\circ} \mathrm{C}$ until use.

2.8. Statistical Analysis. Statistical analyses were performed using the $T$-test after testing for normality using the Shapiro-Wilk test. Values are expressed as means \pm standard deviation. SPSS software (ver. 21.0; IBM Corp., Armonk, NY, USA) was used for all analyses, and statistical significance was defined as $P<0.05$.

\section{Results}

TEM demonstrated that the electron-dense particles accumulated in vesicles within the cells of multiple areas of the brain in rats from the $\mathrm{PM}_{2.5}$ group, an observation which suggests endocytosis of $\mathrm{PM}_{2.5}$ (Figure 2).

The mRNA expression levels of CYP1A1, CYP1B1, iNOS, and $A h r$ were higher in the $\mathrm{PM}_{2.5}$ group compared with the saline group (Figure 3 ). The mRNA levels of CYP1A1 in the ipsilateral temporal cortex in the $\mathrm{PM}_{2.5}$ group were 2.02 $(\mathrm{SD}=0.95)$ fold higher compared with those in the saline group ( $P=0.05$ in $T$-test). The levels of CYP1A1 mRNA in the contralateral temporal cortex in the $\mathrm{PM}_{2.5}$ group were not significantly different from those in the saline group $(P=0.126$ in $T$-test). The levels of $C Y P 1 B 1 \mathrm{mRNA}$ in the ipsilateral and contralateral temporal cortices in the $\mathrm{PM}_{2.5}$ group were $1.81(\mathrm{SD}=0.49)$ and $1.38(\mathrm{SD}=0.33)$ fold higher than those in the saline group, respectively $(P<0.001$ and $P$ $=0.002$ in $T$-test). Levels of $i N O S$ mRNA in the ipsilateral and contralateral temporal cortices in the $\mathrm{PM}_{2.5}$ group were $3.15(\mathrm{SD}=1.98)$ and $2.29(\mathrm{SD}=1.12)$ fold higher compared with those in the saline group $(P=0.037$ and $P=0.48$ in $T$ test).

The mRNA levels of $A h R$ in the ipsilateral temporal cortex in the $\mathrm{PM}_{2.5}$ group were $1.48(\mathrm{SD}=0.42)$ fold higher compared with those in the saline group $(P=0.05$ in T-test), however, they were not significantly different in the contralateral temporal cortex $(P=0.374$ in $T$-test $)$. Upon immunofluorescence examination, the levels of the AhR protein in the ipsilateral primary temporal cortex were increased in the $\mathrm{PM}_{2.5}$ group compared to the saline group (Figure 4).

The abundance of MMP9 and brevican proteins were 2.02 (SD = 0.77; $P=0.02$ in $T$-test) and 2.02 (SD = 0.77; $P$ $=0.02$ in $T$-test) fold higher in the ipsilateral temporal cortex of the $\mathrm{PM}_{2.5}$ group compared with the saline group, respectively (Figure 5).

Detection of RAGE protein levels using ELISA demonstrated an elevation in the $\mathrm{PM}_{2.5}$ group compared with the saline group. The levels of RAGE protein in the ipsilateral temporal cortex of the $\mathrm{PM}_{2.5}$ group were $1.29(\mathrm{SD}=0.05)$ fold higher compared with those in the saline group $(P<0.001$ in $T$-test $)$.

\section{Discussion}

The intracranial injection of $\mathrm{PM}_{2.5}$ increased the levels of AhR in the primary temporal cortex. These increases might be linked with the increased expression levels of oxidative stress molecules (e.g., CYP1A1, CYP1B1, and $i N O S)$. In addition, an increase in the abundance of AhR was accompanied by an increase in the amount of MMP9, a molecule which has been suggested to activate RAGE. These effects of $\mathrm{PM}_{2.5}$ were evident by direct intracellular penetration of nanoparticles as well as by indirect pathways through systemic circulation or paracrine effects.

The impact of $\mathrm{PM}_{2.5}$ on the temporal cortex was triggered by AhR activation. A number of previous studies have suggested that PM trigger proinflammatory reactions triggered by AhR-dependent pathway(s) $[24,25]$. In a 3D culture study, which imitated the alveolar-capillary barrier, diesel exhaust particle exposure led to the upregulation of AhR-regulated genes including $C Y P 1 B 1$ and proinflammatory molecules including $M M P 1$; these responses were inhibited by an AhR antagonist [24]. AhR activation might be involved in the proinflammation responses within the CNS like those occurring in the respiratory system. In 


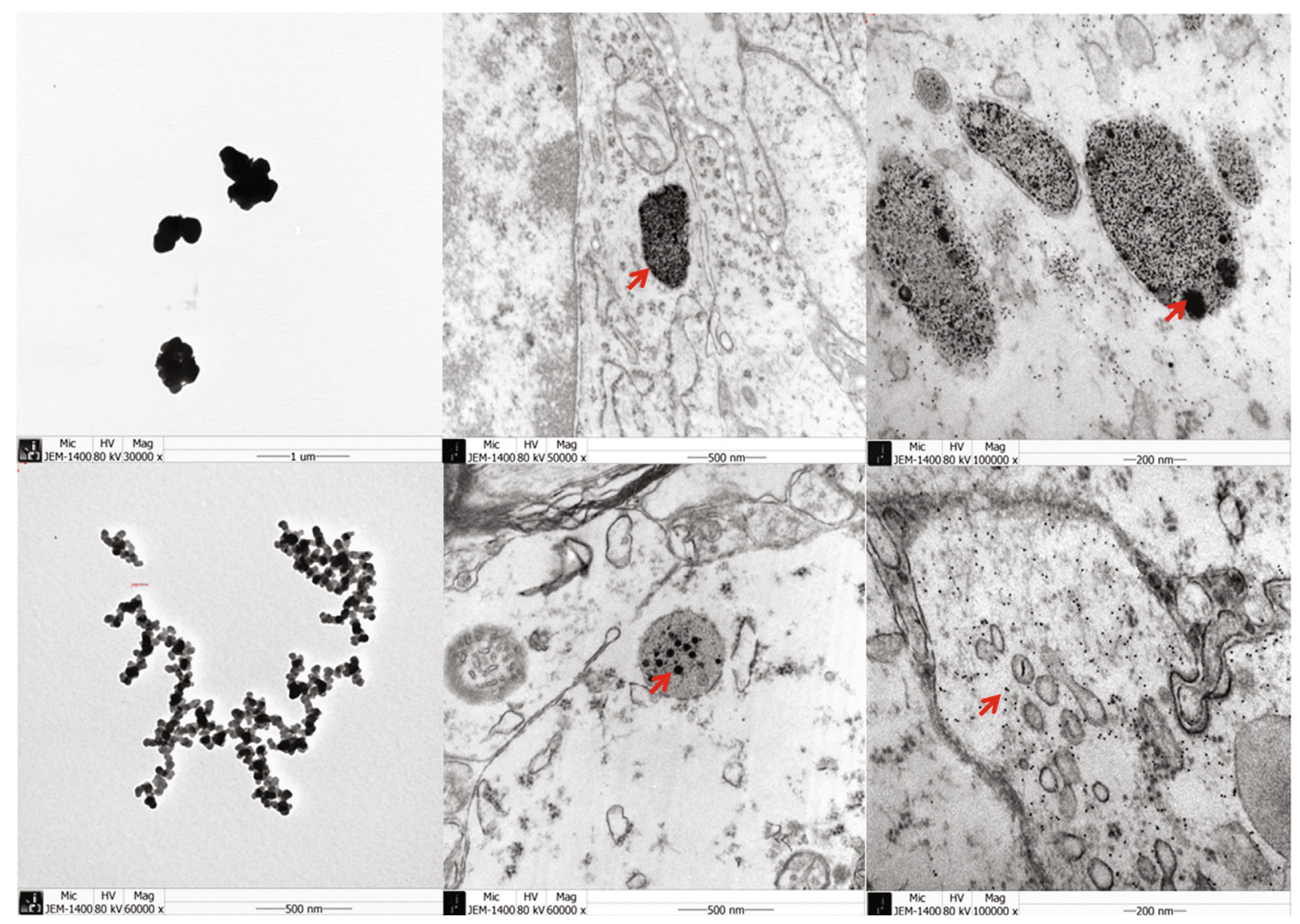

FIgURE 2: Transmission electron microscopy examination of injected brain tissue. The electron-dense particles (red arrow) accumulated in vesicles within the cells of the brain in rats from the $\mathrm{PM}_{2.5}$ group.

lipopolysaccharide-activated microglia, the expression of tumor necrosis factor-alpha, iNOS, and CYP1A1 was increased and reduced following the knockdown of $A h R$ expression in the cerebral cortex of mice [25]. Therefore, it could be presumed that AhR activation may have a crucial role for inducing $\mathrm{PM}_{2.5}$-mediated inflammatory responses in cerebral cortical areas.

In the current study, the levels of key oxidative stress molecules (i.e., CYP1A1, CYP1B1, and iNOS) increased following exposure to $\mathrm{PM}_{2.5}$. Many genes, including CYP1A1, $C Y P 1 B 1$, and proinflammatory genes (e.g., nuclear factor$\mathrm{kB})$, have $A h R$-response elements in their promoters; thus, $A h R$ activation might promote transcription of these genes $[15,26]$. AhR could induce an inflammatory response through these $A h R$-response gene cascades, specifically CYPs, which are important oxidizing enzymes capable of metabolizing xenobiotic compounds [27]. Thus, organic compounds found in PMs could be metabolized by CYPs and produce reactive oxygen species, which in turn may trigger inflammation [24]. In an in vitro study, activation of the AhR/CYP1A1 pathway in cortical neurons and glial cells was described following exposure to the xenobiotic compound Di-(2-ethylhexyl) phthalate [28]. Although increases in the levels of CYP1A1 and $A h R$ in the temporal cortex were localized to the side of injection, the expres- sion of CYP1B1 and $i N O S$ was elevated on both sides of the temporal cortex in the $\mathrm{PM}_{2.5}$ group. Indirect routes of $\mathrm{PM}_{2.5}$ exposure (e.g., cerebrovascular or cerebrospinal fluid circulation) could mediate the increase of these oxidative markers on both sides of the temporal cortex.

Here, it is reported that a $\mathrm{PM}_{2.5}$-mediated increase in $A h R$ levels accompanied an upregulation of MMP9, brevican, and RAGE. MMP9 is a proinflammatory molecule involved in extracellular matrix (ECM) remodeling via degradation of ECM molecules. Work by this group and others have also reported increases in the expression of MMPs following exposure to PM $[5,29,30]$. The observed increase in levels of RAGE in this and several previous studies could be linked with MMP9 upregulation [31, 32]. MMP9 is suggested to mediate an inflammatory response in the CNS by activation of RAGE [33]. In addition to MMP9, AhR activation has been shown to modulate the expression of ECM proteins [34, 35]. The disruption and remodeling of ECM could contribute to the intracellular penetration of PMs and the associated inflammatory responses and fibrosis. In a study in prostate cancer cell lines, MMP9 expression increased after aryl hydrocarbon exposure, events which were suggested to facilitate tumor invasion [36]. In bronchial epithelial cells, AhR agonists led to the upregulation of MMP2, MMP9, 

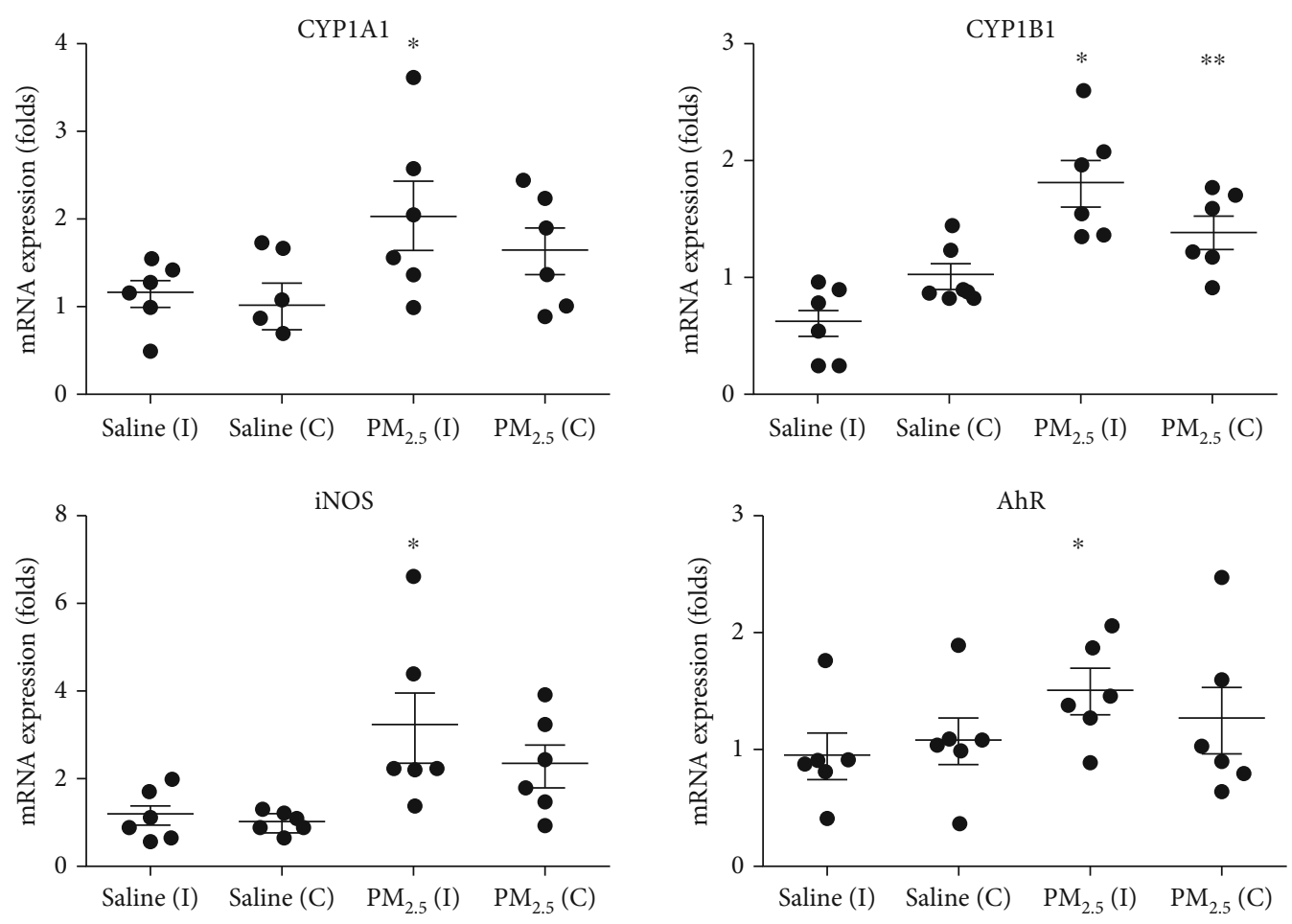

FIGURE 3: Comparisons of the mRNA expression levels of cytochrome P450 1A1 (CYP1A1), CYP1B1, inducible nitric oxide synthase (iNOS), and aryl hydrocarbon receptor $(A h r)$ between saline and $\mathrm{PM}_{2.5}$ groups. The $\mathrm{PM}_{2.5}$ group showed higher mRNA expression levels of $C Y P 1 A 1$, CYP1B1, iNOS, and Ahr in the ipsilateral side of $\mathrm{PM}_{2.5}$ injections. $\left({ }^{*} P<0.05 T\right.$-test between ipsilateral saline vs. ipsilateral $\mathrm{PM}_{2.5}{ }^{* *} \mathrm{P}<0.05 T$ -test between contralateral saline vs. contralateral $\mathrm{PM}_{2.5}$, I: ipsilateral; C: contralateral).
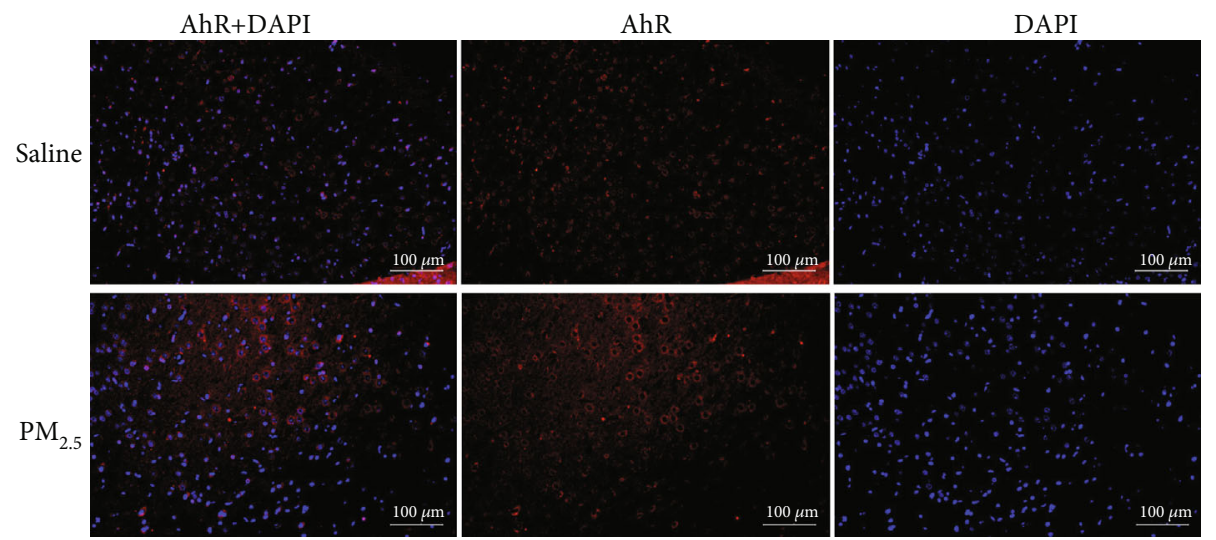

FIGURE 4: Immunofluorescence examination of AhR expressions. The levels of the AhR protein (red) in the ipsilateral primary temporal cortex were increased in the $\mathrm{PM}_{2.5}$ group compared to the saline group (blue: DAPI).

and MMP1 and proposed to contribute to airway remodeling in asthma and chronic obstructive pulmonary disease [37]. Chronic PM exposure altered perineuronal nets in previous studies $[4,5]$. The present study demonstrated an increase of brevican expression after $\mathrm{PM}_{2.5}$ exposures. Brevican is a component of perineuronal nets in the CNS, structures which regulate interneuronal and synaptic plasticity [38]. Therefore, alterations in the ECM via the AhR/MMP9 pathway might also have an effect on the CNS.
Although the present study demonstrated in vivo effects of $\mathrm{PM}_{2.5}$ on the cerebral cortex, further studies to delineate the components of $\mathrm{PM}_{2.5}$ which exert harmful effects and the specific cell types (i.e., neurons and glia) on which these effects are conferred are warranted. Due to the limitation of loading volume for intracranial injection, this study could not delineate the dose-response relations between the exposed $\mathrm{PM}_{2.5}$ concentrations and the cerebral cortical changes. A few previous studies reported the dose-response associations between PM dose and the 


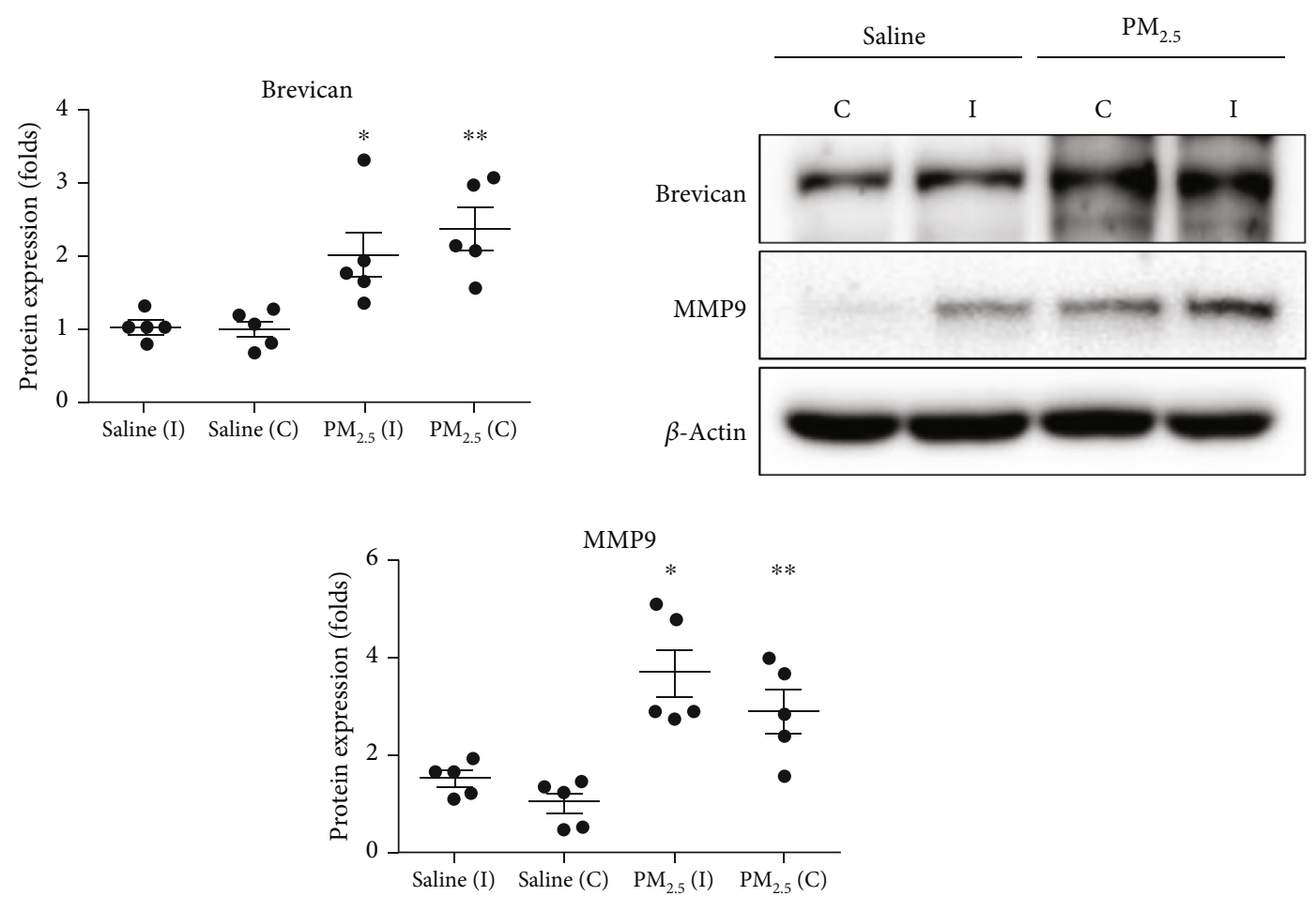

(a)

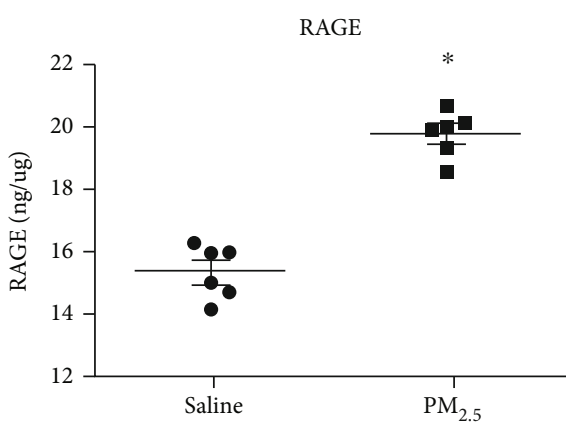

(b)

Figure 5: Comparisons of the protein expression levels of brevican, MMP9, and RAGE. (a) In western blotting, the PM ${ }_{2.5}$ group showed higher protein expression levels of brevican and MMP9 in the ipsilateral side of $\mathrm{PM}_{2.5}$ injections. (b) Enzyme-linked immunosorbent assay showed a higher expression level of advanced glycation end products (RAGE) in the $\mathrm{PM}_{2.5}$ group $\left({ }^{*} P<0.05 T\right.$-test between ipsilateral saline vs. ipsilateral $\mathrm{PM}_{2.5},{ }^{* *} \mathrm{P}<0.05 T$-test between contralateral saline vs. contralateral $\mathrm{PM}_{2.5}$, I: ipsilateral; C: contralateral).

inflammatory changes in brain regions $[39,40]$. In addition, the functional implications of $\mathrm{PM}_{2.5}$ need to be further investigated to better elucidate clinically relevant applications.

\section{Conclusion}

Following intracranial injection, $\mathrm{PM}_{2.5}$ accumulated in the barrier-disrupted cerebral cortex. $\mathrm{PM}_{2.5}$ exposure led to an increase in the levels of AhR, which mediated oxidative stress responses and RAGE-linked inflammation processes in the temporal cortex. The upregulation of MMP9 and brevican might induce ECM remodeling after $\mathrm{PM}_{2.5}$ exposure.

\section{Data Availability}

The raw data of experiments used to support the findings of this study are available from the corresponding author upon request.

\section{Disclosure}

The funders had no role in the design of the study; in the collection, analyses, or interpretation of data; in the writing of the manuscript, or in the decision to publish the results.

\section{Conflicts of Interest}

The authors declare no conflict of interest. 


\section{Authors' Contributions}

SYK did the conceptualization. DL, SML, SP, BSS, and MKP did the investigation. SYK and DL did the methodology. SYK and MKP wrote the original draft. SYK, BSS, and MKP wrote review and editing. All authors have read and agreed to the published version of the manuscript.

\section{Acknowledgments}

This research was supported by the Basic Science Research Program through the National Research Foundation of Korea (NRF) funded by the Ministry of Science, ICT \& Future Planning (NRF-2019R1A2C1089986 and 2020R1A2C4002594).

\section{References}

[1] M. C. Power, A. P. Lamichhane, D. Liao et al., "The association of long-term exposure to particulate matter air pollution with brain MRI findings: the ARIC study," Environ Health Perspect, vol. 126, no. 2, article 027009, 2018.

[2] C.-H. Shih, J.-K. Chen, L.-W. Kuo et al., "Chronic pulmonary exposure to traffic-related fine particulate matter causes brain impairment in adult rats," Particle and Fibre Toxicology, vol. 15 , no. 1 , p. $44,2018$.

[3] X. Liu, X. Qian, J. Xing et al., "Particulate matter triggers depressive-like response associated with modulation of inflammatory cytokine homeostasis and brain-derived neurotrophic factor Signaling pathway in mice," Toxicological Sciences, vol. 164, no. 1, pp. 278-288, 2018.

[4] S. Y. Kim, J. K. Kim, S. H. Park et al., "Effects of inhaled particulate matter on the central nervous system in mice," Neurotoxicology, vol. 67, pp. 169-177, 2018.

[5] S. Y. Kim, D.-h. Lee, S. Park et al., "Neuronal and perineuronal changes of cerebral cortex after exposure to inhaled particulate matter," Scientific Reports, vol. 9, no. 1, article 19421, 2019.

[6] L. G. Costa, T. B. Cole, K. Dao, Y. C. Chang, J. Coburn, and J. M. Garrick, "Effects of air pollution on the nervous system and its possible role in neurodevelopmental and neurodegenerative disorders," Pharmacology \& Therapeutics, vol. 210, article 107523, 2020.

[7] R. G. Lucchini, D. C. Dorman, A. Elder, and B. Veronesi, "Neurological impacts from inhalation of pollutants and the nose-brain connection," Neurotoxicology, vol. 33, no. 4, pp. 838-841, 2012.

[8] G. J. M. Garcia, J. D. Schroeter, and J. S. Kimbell, "Olfactory deposition of inhaled nanoparticles in humans," Inhalation Toxicology, vol. 27, no. 8, pp. 394-403, 2015.

[9] G. Oberdörster, Z. Sharp, V. Atudorei et al., "Translocation of inhaled ultrafine particles to the brain," Inhalation Toxicology, vol. 16, no. 6-7, pp. 437-445, 2004.

[10] A. Peters, B. Veronesi, L. Calderón-Garcidueñas et al., "Translocation and potential neurological effects of fine and ultrafine particles a critical update," Particle and Fibre Toxicology, vol. 3, no. 1, p. 13, 2006

[11] S. B. Lee, J. H. Kim, M. H. Cho, E. S. Choe, K. S. Kim, and S. M. Shim, "Impact of commercial cigarette smoke condensate on brain tissue co-cultured with astrocytes and blood-brain barrier endothelial cells," Journal of Toxicology and Environmental Health. Part A, vol. 80, no. 10-12, pp. 533-541, 2017.
[12] H. S. Jang, J. E. Lee, C. H. Myung, J. I. Park, C. S. Jo, and J. S. Hwang, "Particulate matter-induced aryl hydrocarbon receptor regulates autophagy in keratinocytes," Biomolecules \& Therapeutics, vol. 27, no. 6, pp. 570-576, 2019.

[13] M. van Voorhis, S. Knopp, W. Julliard et al., "Exposure to atmospheric particulate matter enhances Th17 polarization through the aryl hydrocarbon receptor," PLoS One, vol. 8, no. 12, article e82545, 2013.

[14] Q. Ma and K. T. Baldwin, "2,3,7,8-tetrachlorodibenzo-pdioxin-induced degradation of aryl hydrocarbon receptor (AhR) by the ubiquitin-proteasome pathway," The Journal of Biological Chemistry, vol. 275, no. 12, pp. 8432-8438, 2000.

[15] Q. Ma, A. J. Renzelli, K. T. Baldwin, and J. M. Antonini, "Superinduction ofCYP1A1Gene Expression," The Journal of Biological Chemistry, vol. 275, no. 17, pp. 12676-12683, 2000.

[16] Z. Chen, N. Ji, Z. Wang et al., "Fine particulate matter (PM2.5) promoted the invasion of lung cancer cells via an ARNT2/PP2A/STAT3/MMP2 pathway," Journal of Biomedical Nanotechnology, vol. 14, no. 12, pp. 2172-2184, 2018.

[17] C. Yue, C. Ji, H. Zhang et al., "Protective effects of folic acid on PM2.5-induced cardiac developmental toxicity in zebrafish embryos by targeting $\mathrm{AhR}$ and $\mathrm{Wnt} / \beta$-catenin signal pathways," Environmental Toxicology, vol. 32, no. 10, pp. 23162322, 2017.

[18] A. M. Espinosa-Oliva, R. M. de Pablos, and A. J. Herrera, "Intracranial injection of LPS in rat as animal model of neuroinflammation," Methods in Molecular Biology, vol. 1041, pp. 295-305, 2013.

[19] J. D. Finan, F. S. Cho, S. G. Kernie, and B. Morrison III, "Intracerebroventricular administration of chondroitinase $\mathrm{ABC}$ reduces acute edema after traumatic brain injury in mice," BMC Res Notes, vol. 9, no. 1, p. 160, 2016.

[20] M. H. Hettiaratchi, M. J. O'Meara, C. J. Teal, S. L. Payne, A. J. Pickering, and M. S. Shoichet, "Local delivery of stabilized chondroitinase $\mathrm{ABC}$ degrades chondroitin sulfate proteoglycans in stroke-injured rat brains," Journal of Controlled Release, vol. 297, pp. 14-25, 2019.

[21] S. Yang, S. Hilton, J. N. Alves et al., "Antibody recognizing 4sulfated chondroitin sulfate proteoglycans restores memory in tauopathy-induced neurodegeneration," Neurobiology of Aging, vol. 59, pp. 197-209, 2017.

[22] S. K. Park, Y. M. Jeon, B. S. Son, H. S. Youn, and M. Y. Lee, "Proteomic analysis of the differentially expressed proteins by airborne nanoparticles," Journal of Applied Toxicology, vol. 31, no. 5, pp. 463-470, 2011.

[23] S.-P. Jin, Z. Li, E. K. Choi et al., "Urban particulate matter in air pollution penetrates into the barrier- disrupted skin and produces ROS-dependent cutaneous inflammatory response _in vivo_," Journal of Dermatological Science, vol. 91, no. 2, pp. 175-183, 2018.

[24] B. C. Brinchmann, T. Skuland, M. H. Rambøl et al., "Lipophilic components of diesel exhaust particles induce proinflammatory responses in human endothelial cells through AhR dependent pathway(s)," Particle and Fibre Toxicology, vol. 15, no. 1, p. 21, 2018.

[25] Y. H. Lee, C. H. Lin, P. C. Hsu et al., "Aryl hydrocarbon receptor mediates both proinflammatory and anti-inflammatory effects in lipopolysaccharide-activated microglia," Glia, vol. 63, no. 7, pp. 1138-1154, 2015.

[26] C. F. A. Vogel and F. Matsumura, "A new cross-talk between the aryl hydrocarbon receptor and RelB, a member of the 
NF- $\kappa$ B family," Biochemical Pharmacology, vol. 77, no. 4, pp. 734-745, 2009.

[27] M. R. Namazi, "Cytochrome-P450 enzymes and autoimmunity: expansion of the relationship and introduction of free radicals as the link," Journal of Autoimmune Diseases, vol. 6, no. $1,2009$.

[28] A. K. Wojtowicz, A. M. Sitarz-Glownia, M. Szczesna, and K. A. Szychowski, "The action of di-(2-ethylhexyl) phthalate (DEHP) in mouse cerebral cells involves an impairment in aryl hydrocarbon receptor (AhR) signaling," Neurotoxicity Research, vol. 35, no. 1, pp. 183-195, 2019.

[29] X. Han, H. Liu, Z. Zhang et al., "Epitranscriptomic 5methylcytosine profile in PM2.5-induced mouse pulmonary fibrosis," Genomics, Proteomics \& Bioinformatics, vol. 18, no. 1, pp. 41-51, 2020.

[30] P. Li, J. Wang, F. Guo, B. Zheng, and X. Zhang, "A novel inhibitory role of microRNA-224 in particulate matter 2.5-induced asthmatic mice by inhibiting TLR2," Journal of Cellular and Molecular Medicine, vol. 24, no. 5, pp. 3040-3052, 2020.

[31] D. B. Barton, B. C. Betteridge, T. D. Earley, C. S. Curtis, A. B. Robinson, and P. R. Reynolds, "Primary alveolar macrophages exposed to diesel particulate matter increase RAGE expression and activate RAGE signaling," Cell and Tissue Research, vol. 358, no. 1, pp. 229-238, 2014.

[32] W. Zou, F. He, S. Liu et al., "PM2.5 induced the expression of fibrogenic mediators via HMGB1-RAGE signaling in human airway epithelial cells," Canadian Respiratory Journal, vol. 2018, Article ID 1817398, 10 pages, 2018.

[33] D. Dwir, B. Giangreco, L. Xin et al., "MMP9/RAGE pathway overactivation mediates redox dysregulation and neuroinflammation, leading to inhibitory/excitatory imbalance: a reverse translation study in schizophrenia patients," Molecular Psychiatry, vol. 25, no. 11, pp. 2889-2904, 2020.

[34] E. A. Andreasen, L. K. Mathew, and R. L. Tanguay, "Regenerative growth is impacted by TCDD: gene expression analysis reveals extracellular matrix modulation," Toxicological Sciences, vol. 92, no. 1, pp. 254-269, 2006.

[35] E. A. Andreasen, L. K. Mathew, C. V. Lohr, R. Hasson, and R. L. Tanguay, "Aryl hydrocarbon receptor activation impairs extracellular matrix remodeling during zebra fish fin regeneration," Toxicological Sciences, vol. 95, no. 1, pp. 215-226, 2007.

[36] M. Haque, J. Francis, and I. Sehgal, "Aryl hydrocarbon exposure induces expression of MMP-9 in human prostate cancer cell lines," Cancer Letters, vol. 225, no. 1, pp. 159-166, 2005.

[37] M. J. Tsai, Y. L. Hsu, T. N. Wang et al., “Aryl hydrocarbon receptor (AhR) agonists increase airway epithelial matrix metalloproteinase activity," Journal of Molecular Medicine (Berlin, Germany), vol. 92, no. 6, pp. 615-628, 2014.

[38] R. Frischknecht and C. I. Seidenbecher, "Brevican: a key proteoglycan in the perisynaptic extracellular matrix of the brain," The International Journal of Biochemistry \& Cell Biology, vol. 44, no. 7, pp. 1051-1054, 2012.

[39] S. Hajipour, Y. Farbood, M. K. Gharib-Naseri et al., "Exposure to ambient dusty particulate matter impairs spatial memory and hippocampal LTP by increasing brain inflammation and oxidative stress in rats," Life Sciences, vol. 242, p. 117210, 2020.

[40] Y. Shou, X. Zhu, D. Zhu et al., “Ambient PM2.5 chronic exposure leads to cognitive decline in mice: from pulmonary to neuronal inflammation," Toxicology Letters, vol. 331, pp. $208-217,2020$. 